\title{
A structural UGDH variant associated with standard Munchkin cats
}

\author{
Ann-Kathrin Struck', Marina Braun ${ }^{1}$, Kim Aline Detering ${ }^{1}$, Peter Dziallas², Jasmin Neßler², Michael Fehr², \\ Julia Metzger ${ }^{1}$ and Ottmar Distl ${ }^{1 *}$ (D)
}

\begin{abstract}
Background: Munchkin cats were founded on a naturally occurring mutation segregating into long-legged and short-legged types. Short-legged cats showed disproportionate dwarfism (chondrodysplasia) in which all four legs are short and are referred as standard Munchkin cats. Long-legged animals are referred as non-standard Munchkin cats. A previous study using genome-wide single nucleotide polymorphisms (SNPs) for genome-wide association analysis identified a significantly associated region at 168-184 Mb on feline chromosome (FCA) B1.

Results: In this study, we validated the critical region on FCA B1 using a case-control study with 89 cats and 14 FCA B1-SNPs. A structural variant within UGDH (NC_018726.2:g.173294289_173297592delins108, Felis catus 8.0, equivalent to NC_018726.3:g.174882895_174886198delins108, Felis catus 9.0) on FCA B1 was perfectly associated with the phenotype of short-legged standard Munchkin cats.

Conclusion: This UGDH structural variant very likely causes the chondrodysplastic (standard) phenotype in Munchkin cats. The lack of homozygous mutant phenotypes and reduced litter sizes in standard Munchkin cats suggest an autosomal recessive lethal trait in the homozygote state. We propose an autosomal dominant mode of inheritance for the chondrodysplastic condition in Munchkin cats.
\end{abstract}

Keywords: Munchkin, Cat, Felis catus, Chondrodysplasia, Whole genome sequencing, UGDH

\section{Background}

Short-legged cats have been reported as early as 1944, describing individuals with unusually short forelimbs but long-legged hind limbs, resulting in forelimb micromelia [1-3]. Today's Munchkin cats presumably have their roots in a female cat with all four short legs on which Sandra Hockenedel [4] used to found the breed in 1983. This short-legged animal gave birth to a healthy litter, with half of the kittens showing short legs. This shortlegged female cat and one of its short-legged male kittens established today's Munchkin cat breed. To expand the Munchkin cats' gene pool, these two Munchkin cats were mated to long-legged cats. Through this outcross

\footnotetext{
* Correspondence: ottmar.distl@tiho-hannover.de

'Institute of Animal Breeding and Genetics, University of Veterinary Medicine Hannover (Foundation), 30559 Hannover, Germany

Full list of author information is available at the end of the article
}

program, a variety of coat colors and patterns as well as different Munchkin cat subtypes were developed [4-10] (Additional file 1). Breeders refer to short-legged as standard Munchkin and long-legged as non-standard Munchkin cats [4]. Offspring from standard Munchkin cat parents can segregate into standard and nonstandard Munchkin cats. Munchkin cats have been recognized by The International Cat Association (TICA) as an independent breed since 1994. Breeding data collected by TICA suggest a dominant mode of inheritance $[11,12]$ and lethality in the homozygous state at an early embryonic stage [5].

The Munchkin cat phenotype and genotype is not yet fully characterized. A genome-wide association study (GWAS) was performed for the standard Munchkin phenotype resulting in a highly significant critical region at $168-184 \mathrm{Mb}$ on feline chromosome (FCA) B1 [13].

(c) The Author(s). 2020 Open Access This article is licensed under a Creative Commons Attribution 4.0 International License, which permits use, sharing, adaptation, distribution and reproduction in any medium or format, as long as you give appropriate credit to the original author(s) and the source, provide a link to the Creative Commons licence, and indicate if changes were made. The images or other third party material in this article are included in the article's Creative Commons licence, unless indicated otherwise in a credit line to the material. If material is not included in the article's Creative Commons licence and your intended use is not permitted by statutory regulation or exceeds the permitted use, you will need to obtain permission directly from the copyright holder. To view a copy of this licence, visit http://creativecommons.org/licenses/by/4.0/. The Creative Commons Public Domain Dedication waiver (http://creativecommons.org/publicdomain/zero/1.0/) applies to the data made available in this article, unless otherwise stated in a credit line to the data. 
The present study characterized the standard Munchkin phenotype using computed tomography (CT) scans and comparative long bone measurements. Pedigree data of Munchkin cats were collated to establish the mode of inheritance. In order to unravel the responsible mutation for standard Munchkin cats, we analyzed whole genome sequencing (WGS) data of a Munchkin cat family and controls for Munchkin-associated variants. Candidate variants were validated in large cohorts of standard and non-standard Munchkin cats as well as in a cat breed panel with the most popular breeds. We identified a structural UDP-glucose 6-dehydrogenase $(U G D H)$ variant perfectly segregating with the standard Munchkin cat phenotype. For this variant, we assumed dominance as homozygous mutants were not observed.

\section{Results}

\section{Munchkin cat phenotype}

The standard Munchkin cats of the subtype Genetta showed a disproportionate short stature with shortened fore- and hind limbs compared to a female non-standard

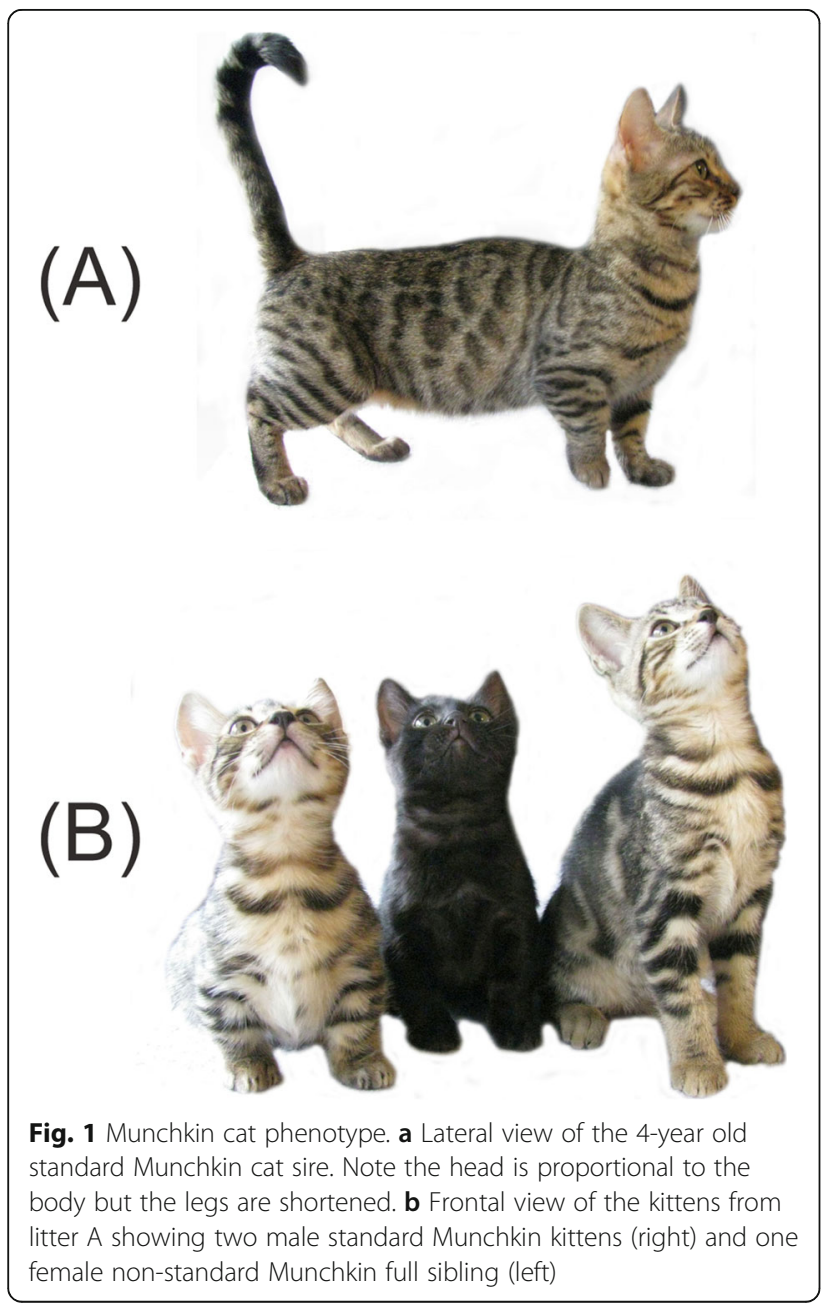

Munchkin cat (Fig. 1). Subtype Genetta resulted from the outcrossing program of standard Munchkin with Bengal cats and this subtype is recognized by TICA as an experimental breed. The upper- and forearms of the front limbs as well as the upper- and forelegs had nearly the same length in the standard Munchkin cats. The size of paws was proportional to the body. The head of both types, standard and non-standard Munchkin cats showed no signs of chondrodysplastic changes such as brachygnathia superior or a spherical appearance.

CT of the forelimbs of the 4-year old standard Munchkin tomcat showed a shortening of all distal und proximal long bones including humerus, radius, ulna and metacarpalia (Fig. 2 and Additional file 2). Lengths of the humerus, radius, ulna, metacarpalia, femur and tibia of the standard Munchkin cat were reduced by 71,58 , $64,84,74$, and $70 \%$, respectively, in comparison to the domestic cat. Metatarsalia had normal length. All bones of the front and hind limbs exhibited higher average diaphyseal diameters, in particular humerus $(+14 \%)$ and femur (+29\%). Furthermore, the humerus showed a slight internal rotation along its longitudinal axis, resulting in a moderate incongruity in the elbow joint with axial deviation. The humerus compacta in the middle segment revealed a moderate degree of thickening of 2.4 $\mathrm{mm}$ (0.09 in). Furthermore, the radius was too short in relation to the ulna, as well as the ulna was slightly medially rotated, whereas the radius was bent to a high degree in the longitudinal axis of about $43^{\circ}$. This resulted in an incongruity in the ulnocarpal and radiocarpal joint. The hind limbs did not show any rotation and structural effects.

\section{Pedigree analysis}

Pedigree analysis of the Munchkin cat family of the subtype Genetta demonstrated that three repeated matings between a standard Munchkin tomcat and a standard Munchkin queen resulted in seven standard Munchkin and two non-standard Munchkin cats (Additional file 3). As reported by breeders, matings among standard Munchkin cats resulted in reduced litter sizes.

\section{Association and haplotype analysis}

Association analysis for 14 SNPs on FCA B1 gave significant $P$-values for $9 / 14$ SNPs (Additional file 4). These nine significant SNPs were located in the critical region FCA B1. The SNPs B1_9 (g.173759872A > G) and B1_10 (g.174407393 $\mathrm{T}>\mathrm{C}$ ) reached the highest $\mathrm{P}$-values for both the genotype (B1_9, $P=9.17^{-18} ; \mathrm{B} 1 \_10, \quad P=$ $5.79^{-18}$ ) and allele (B1_9, $P=7.59^{-17} ; \mathrm{B} 1 \_10, \quad P=$ $6.33^{-17}$ ) distribution among cases and controls. Haplotype analysis corroborated the significant association for the single SNPs (Additional file 5). 
(A)

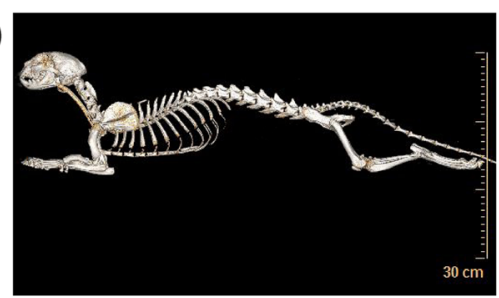

(C)

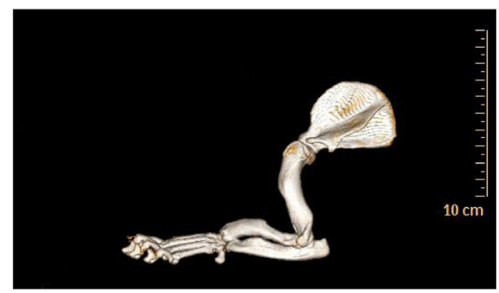

(E)

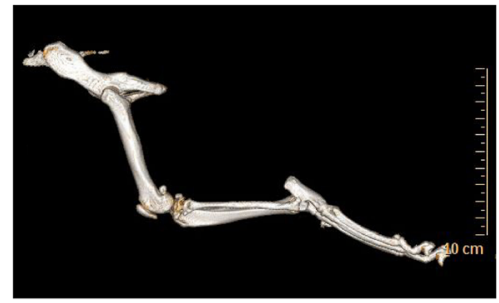

(B)

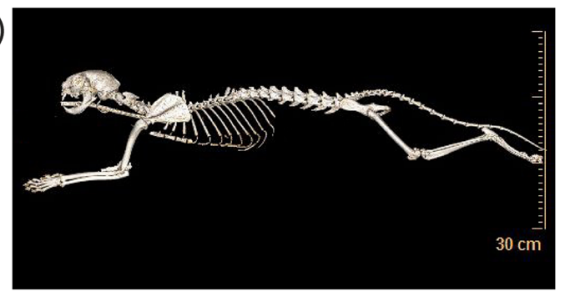

(D)

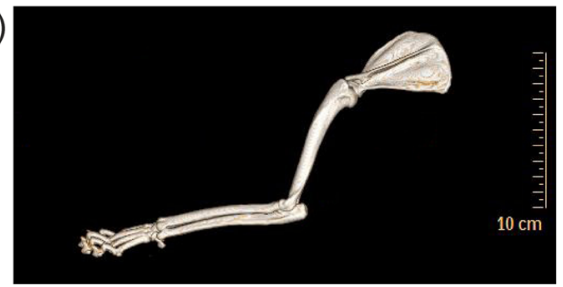

(F)

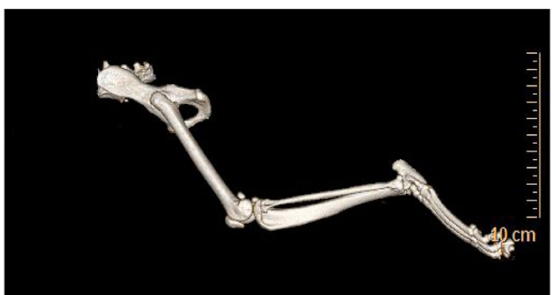

Fig. 2 Computed tomography of a 4-year old standard Munchkin cat sire and an adult female domestic cat control. Lateral view of a standard Munchkin cat $(\mathbf{a}, \mathbf{c}, \mathbf{e})$ and a domestic cat $(\mathbf{b}, \mathbf{d}, \mathbf{f})$. The limbs of the standard Munchkin cat are shortened in relation to the body. The fore limbs of the standard Munchkin cat (c) show a shortening of all distal and proximal long bones and higher diaphyseal diameters, particularly of humerus, compared to the fore limbs of domestic cat (d). The hind limbs (e, f) also show higher diaphyseal diameters in particular in the femur as well as a shortening of the long bones

Whole genome sequencing and variant detection

A mean read coverage of 11.99X (non-standard Munchkin kitten), 12.67X (standard Munchkin kitten), 12.90X (standard Munchkin sire) and 8.49X (standard Munchkin dam) was obtained in WGS data. A filter analysis for exclusively heterozygous genotypes in the standard Munchkin cats and homozygous wild genotypes in 16 control cats did not reveal functional variants on FCA B1, B4 and X.

Thus, an obvious protein modifying candidate variant was not identified in the genome-wide associated region on FCA B1 at 168 to $184 \mathrm{Mb}$ or within the genomic regions with the next highest peaks on FCA B4 and X from the previous GWAS [13].

Structural variant analysis with LUMPY software revealed one variant on FCA B1 harbouring a heterozygous deletion of $3303 \mathrm{bp}$ in all three standard Munchkin cats but homozygous wild type in the non-standard Munchkin kitten and all 16 controls. The $3303 \mathrm{bp}$ deletion was located within UDP-glucose 6-dehydrogenase (UGDH) at 173,294,289-173, 297,592 bp (Felis catus 8.0; NC_018726.2:g.173294289_ 173297592delins108), equivalent to $174,882,895-174,886$, $198 \mathrm{bp} \quad$ (Felis catus 9.0; NC_018726.3:g.174882895 174886198delins108) on FCA B1 within the critical region.
This $3303 \mathrm{bp}$ deletion comprises $1191 \mathrm{bp}$ of intronic sequences (intron 10-11, ENSFCAT00000009602.6 or intron 9-10, ENSFCAT00000055794.2), exon 11 (ENSFC AT00000009602.6) or 10 (ENSFCAT00000055794.2) and 2001 bp of the 3'-UTR (Additional file 6).

\section{Sanger sequencing}

Sanger cDNA sequencing confirmed the break points of the deletion predicted in the LUMPY software. Furthermore, an insert of $108 \mathrm{bp}$ was identified between the break points of the deletion in all three standard Munchkin cats (5' -AAATCACTAAATCACTAAATAA AAGTTCAAAGATATGAACATAGATATGAATGACT ACCAGAGAAGGAGATTTGCTTCCCGGATTATAG AGAGATTATTTAGAGATTA-'3). An NCBI Blast search (https://blast.ncbi.nlm.nih.gov/Blast.cgi?PAGE_ TYPE=Blast Search) of this insert against feline reference genomes (Felis catus 8.0 and Felis catus 9.0) did not identify any regions with significant sequence homology. However, two sequence similarities (100\%) of $51 \mathrm{bp}$ ( $\max$ score 95.3 , E-value $8 \times 10^{-18)}$ ) were identified on FCA B1. The first one, within $U G D H$ distal to the indel in the exonic region at 173,290,767-173,290,817 bp (Felis catus 8.0 ) and 174,879,349-174,879,399 bp (Felis catus 
9.0) and the second, in the intergenic region of ENSFCAG00000031593 at 134,584,870- $134,584,920 \mathrm{bp}$ (Felis catus 9.0).

\section{Mutation analysis}

We did not detect variants in wild type cats for the cDNA sequence of exon 10 and 11 (ENSFCAT00000009602.6) or exon 9 and 10 (ENSFCAT00000055794.2) and the proximal parts of the 3'UTR using primer pairs within exon 10 and 3'UTR (PCR-type 3 and PCR-type 1, Additional file 7). Amplicons of cDNA from wild type cats were of an expected size of $2514 \mathrm{bp}$ (PCR-type 1) or $185 \mathrm{bp}$ (PCR-type 3). Sanger sequencing of wild type allele cDNA for a region including exon 10 (ENSFCAT00000009602.6) or exon 9 (ENSFCAT00000055794.2) and 3'UTR (PCR-type 4-7) confirmed the 3'UTR region reference genome sequence (Ensembl database).

PCR-type 2 was designed to show whether the $108 \mathrm{bp}$ insert (see above) is transcribed into mRNA and in this case, should give an amplicon size of $110 \mathrm{bp}$ only for the mutant allele in standard Munchkin cats. In standard Munchkin cats heterozygous for the NC_018726.3: g.174882895_174886198delins108 (Felis catus 9.0) mutation, PCR-type 1 should yield two amplicons with sizes of $402 \mathrm{bp}$ (insertion not transcribed) or $510 \mathrm{bp}$ (insertion transcribed) and $2514 \mathrm{bp}$ for the mutant and wild type allele, respectively. The shorter fragment size of $402 \mathrm{bp}$ was calculated based on genomic DNA analysis assuming deletion of exon 11 (ENSFCAT00000009602.6) or exon 10 (ENSFCAT00000055794.2) and $2001 \mathrm{bp}$ of the proximal part of the 3'UTR. However, PCR-type 1 yielded an amplicon size of $493 \mathrm{bp}$ for the mutant allele in standard Munchkin cats. This amplicon contained 73 bp of exon 10 (ENSFCAT00000009602.6) or exon 9 (ENSFCAT00000055794.2) and 309 bp from intron 1011 (ENSFCAT00000009602.6) or intron 9-10 (ENSFCAT00000055794.2) and in addition, $111 \mathrm{bp}$ of the 3'UTR (Fig. 3 and Additional file 8). We found an alternative splice site in the intronic sequence with the motif TACACAatttaaaa explaining the partial intron retention of $309 \mathrm{bp}$. PCR-type 2 failed to generate an amplicon and thus, suggests that the $108 \mathrm{bp}$ insert is not transcribed into mRNA.

Open reading frame finder (ORF, http://www.ncbi. nlm.nih.gov/projects/gorf/) prediction for the mutated cDNA sequence predicted an UGDH protein truncated by 12 aa (Additional file 9). For non-standard and wild type cats, an UGDH wild type protein of 494 aa (UGDH-201, ENSFCAT00000009602.6) and 427 aa (UGDH-202, ENSFCAT00000055794.2) was predicted. The partial intron retention was predicted to result in an elongation of exon 9 (ENSFCAT00000055794.2) or exon 10 (ENSFCAT00000009602.6) by 75 bp with a new stop codon (Additional file 8), as well as a modified 3'UTR sequence starting within the partially retained intronic sequence. The proximal $218 \mathrm{bp}$ of the wild type 3'UTR were found to be missing in the mutated allele according to our cDNA Sanger sequences. Based on Felis catus 9.0, the predicted protein sequence modification (start at aa 459 for ENSFCAT00000009602.6 or aa 392 for ENSFCAT00000055794.2) was found to be located within the UDP-glucose 6-dehydrogenase (PTHR11374: SF35, PANTHER), UDP-glucose dehydrogenase/UDPmannac dehydrogenase (PTHR11374, PANTHER), UDPglc_DH_euk (PIRSF500133, PIRSF) and UDPglc_ GDPman_dh domain (PIRSF000124, PIRSF) (Additional file 10). Comparative species alignments with Clustal Omega (UGDH-201, ENSFCAT00000009602.6) located the indel in a highly conserved region of $U G D H$ (Additional file 11).

\section{Validation}

The $U G D H$ structural variant comprising a deletion and an insertion on FCA B1 was found in all 42 standard Munchkin cats whereas the 15 non-standard Munchkin cats and all 203 cats of other breeds were homozygous wild type. Perfect segregation of the $U G D H$ structural variant with the standard and non-standard Munchkin cats was shown for the Munchkin cat family of the subtype Genetta (Additional file 3).

\section{Discussion}

This is the first study identifying a mutation in $U G D H$ on FCA B1 perfectly associated with standard Munchkin cats exhibiting a disproportionate stature with shortened legs. In our study, we confirmed the significantly associated genome region on FCA B1 at 168-184 Mb [13] in a case-control and haplotype analysis and identified a potential causative structural variant in $U G D H$. UGDH is involved in heparan sulfate proteoglycan (HSPGs) synthesis through catalyzing conversion of UDP-glucose into UDP-glucuronic acid (UDP-GlcA) [14]. Proteoglycans regulate growth factors and morphogens such as fibroblast growth factor (Fgf), hedgehog (Hh), transforming growth factor- $\beta$ (TGFß) and wingless (Wg, Wnt family) [14-17]. Studies on growth related signaling molecules showed Wnt and Fgf proteins necessary for limb structure, ensuring proliferation, differentiation and cell survival $[18,19]$. Various mutations in Wnt proteins (Wnt-3a and Wnt-7a) were found to result in abnormalities during limb formation including irregular limb size $[17,20]$. Furthermore, a disruption of $f g f 10$ in mutant zebrafish (daedalus) was shown to provoke an absence of $f g f 10$ activity and thus, a severely compromised fin bud development [19]. Whether the standard Munchkin cat fits into a model with disturbed proteoglycan signaling and aberrant signals for morphogens requires further 


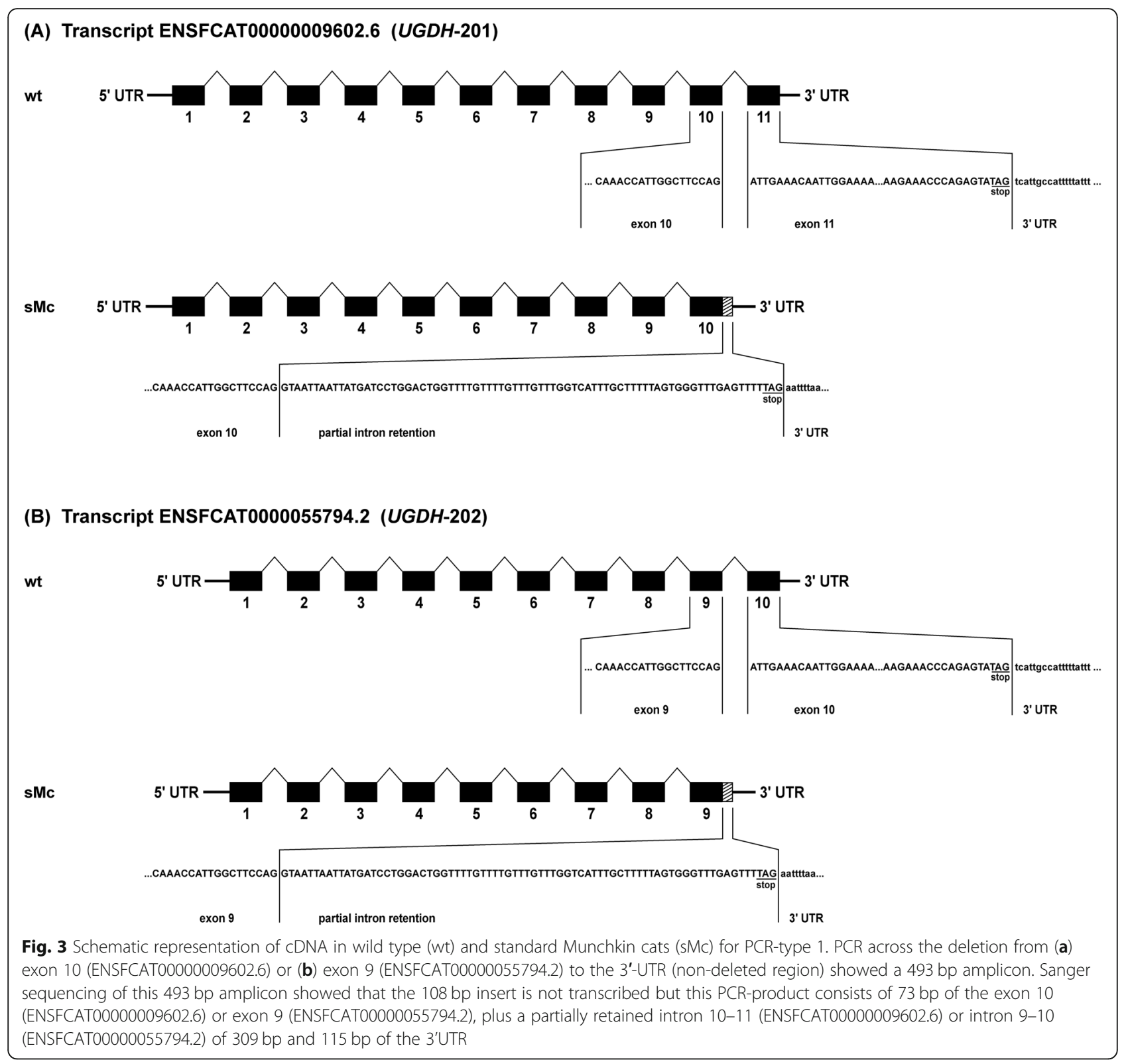

studies. Furthermore, the finding that no homozygous mutant individual was present in the study population supported the previous suggestion of lethality of homozygous mutant individuals at an early embryonic stage [5].

Our investigations of heterozygous mutant standard Munchkin cats compared to homozygous wild type cats showed a partial retention of the last intron in $U G D H$ resulting in a loss of an acceptor site and a new alternative donor site. cDNA sequencing results revealed a partial intron retention of $309 \mathrm{bp}$ as well as a predicted UGDH protein with a truncation of 12 aa and a modification of the aa sequence. Previous studies showed that intron-retaining transcripts are prone to produce novel protein isoforms with modified biological functions resulting in detrimental effects on cells and tissues [2123]. This was also observed in Miniature Zebu, in which an aggrecan (ACAN)-associated mutation caused a recessive lethal chondrodysplasia due to a truncated and modified protein [24]. In Dexter cattle, a functionally null variant of $A C A N$ was found in a homozygous state in spontaneously aborted fetuses and non-viable calves with a bulldog-like phenotype [25]. In our study, we propose the predicted truncation and modification of UGDH as the reason for an early embryonic death in a homozygous mutant state. Studies for $U G D H$ in mice and drosophila (sugarless or kiwi) mutant embryos deficient for UGDH activity showed also embryonic lethality 
[26-30]. The zebrafish ugdh mutant jekyll was found to be lethal due to severe defects during organogenesis of the heart [28]. Heterozygous standard Munchkin exhibit reduced leg length without impaired health, as found in Dexter cattle [25].

\section{Conclusions}

In conclusion, we identified a structural $U G D H$ variant perfectly associated with the standard Munchkin phenotype. No homozygous mutant cats have been observed, supporting the suggestion that the mutation has an autosomal dominant mode of inheritance for the chondrodysplastic condition in Munchkin cats. A diagnostic test is now available for cat breeders to prove if their animals carry the standard Munchkin cat mutation in order to get recognition by TICA.

\section{Methods}

\section{Animals}

Genomic DNA was isolated from EDTA-blood, hair root or tissue samples. We sampled 42 standard Munchkin and 15 non-standard Munchkin cats from several subtypes (Additional file 12). Standard Munchkin cats included the subtypes Genetta $(n=10)$, Napoleon $(n=2)$, Dwelf, $(n=1)$, Bambino $(n=10)$, Lambkin $(n=1)$, longhaired $(n=11)$ and shorthaired $(n=7)$. Non-standard Munchkin cat samples were available for the subtypes Genetta $(n=2)$, Bambino $(n=6)$, longhaired $(n=1)$ and shorthaired $(n=6)$. Cats of the subtype Genetta belonged to a family segregating into long-legged and short-legged types (Additional file 3). The sire and three of his kittens (two standard and one non-standard Munchkin cats) underwent a detailed clinical examination. The parents and two full sibling kittens from this segregating litter were chosen for WGS. For controls, we sampled 203 non-Munchkin cats representing British shorthair, Bengal, Ragdoll, Exotic Shorthair, Don Sphinx, Birman, Chartreux, Maine Coon, Norwegian Forest, Persian, Russian Blue, Scottish Fold, Siamese, Siberian, Turkish Angora, Oriental Shorthair, and domestic shorthair.

\section{Clinical examination}

For the clinical investigation, members of the segregating Munchkin cat family of the subtype Genetta were available. A cat breeder provided a 4-year old standard Munchkin tomcat and three of his progeny including two male standard and a female non-standard Munchkin kitten for a detailed examination. The 4-year old standard Munchkin tomcat underwent a CT scan in sternal recumbency using a multislice helical CT scanner (Brilliance 64-CT, Philips Medical Systems, Best, The Netherlands). A slice thickness for the head of $0.67 \mathrm{~mm}$ (0.026 in) and settings of $120 \mathrm{kV} / 250 \mathrm{mAs}$ and for the body a slice thickness of $0.67 \mathrm{~mm}$ (0.026 in) and settings of $140 \mathrm{kV} / 250 \mathrm{mAs}$ were set for analysis. A $1024 \times 1024$ matrix was used.

In addition, absolute total length, diaphyseal length and the diaphyseal diameter of humerus, radius, ulna, femur, tibia, metacarpalia and metatarsalia bones were measured according to Jones and Jolly [31]. The relative length of individual bones was determined through the ratio of length of the respective individual bone to the length measured from the proximal end of the humerus to the distal end of metarcarpus III.

\section{Haplotype analysis}

To refine the critical genome-wide associated region on FCA B1, Kompetitive Allele Specific PCR (KASP) assay was used (LGC Genomics, Middlesex, UK). We selected 14 SNPs (PRJEB30080) of which four SNPs were located distally to the critical region and ten SNPs within the genome-wide associated region at a distance among each other of about 430 to $7600 \mathrm{~kb}$ on FCA B1. These 14 SNPs were genotyped in 9 standard Munchkin cats, one non-standard Munchkin cat and 79 controls. These animals were unrelated. KASP genotyping was performed according to standard protocols (LGC) using an ABI7300 real-time system for 96 well plates (Additional file 13). A case-control analysis was carried out using SAS/Genetics, version 9.4 (Statistical Analysis System, Cary, NC, USA). Haplotype analysis was done with the procedure HAPLOTYPE in SAS.

\section{Whole genome sequencing}

Animals for WGS were from the Munchkin cat family of the subtype Genetta including the standard Munchkin parents (sire and dam) and two members of their litter with a standard Munchkin cat male and a non-standard Munchkin cat female (Additional file 3). Libraries were prepared using the NEBNext Ultra II DNA Library Prep Kit for Illumina (New England BioLabs, Ipswich, MA, USA) and run on an Illumina NextSeq500 in a $2 \times 150$ bp paired-end mode. Quality control was performed using fastqc 0.11 .5 [32] and reads were trimmed using PRINSEQ (V 0.20.4) [33].

WGS data was mapped to the cat reference genome Felis catus 8.0 (Ensembl) using BWA 0.7.13 [34]. Sorting, duplicate marking and indexing was performed using Picard tools (http://broadinstitute.github.io/picard/, version 2.9.4) and SAMtools 1.3.1 [35]. For final variant calling GATK version 3.7 [36] with the Base Quality Score Recalibrator (BQSR), Haplotype Caller [37] and Variant Recalibrator were used. Variant calling data were compared to WGS data from 16 different individuals including Bengal (SAMN05980341, SAMN05980358), Siamese (SAMN05980342), Peterbald (SAMN05980359, SAMN14103114), Oriental shorthair (SAMN14103123, SAMN14103124, SAMN14103127), and 
domestic shorthair (SAMN14103116, SAMN14103119, SAMN14103120, SAMN14103121, SAMN05980374, SAMN05980340, SAMN05980323, SAMN05980325).

A read depth of 8-999 and quality score values $>20$ were applied for variant detection. Those variants, which were heterozygous in the three standard Munchkin cats, homozygous wild type in the non-standard Munchkin cat and homozygous wild type in all controls and concordant to an autosomal monogenic dominant inheritance, were filtered using SAS (version 9.4, Statistical Analysis System, Cary, NC, USA) for further investigation. Only variants with high or moderate effects according to SNPEff version $4.3 \mathrm{t}$ (2017-11-24, SNPEff database Felis catus 8.0) [38] were considered for further analysis. A candidate gene list from previous studies about chondrodysplasia was used to find candidate genes in the filtered variant list [24].

\section{Structural variant detection}

For structural variant detection, LUMPY software, version 0.2.13 [39], integrating multiple structural variation signals jointly across multiple samples, was run for Bam files of all four Munchkin cats and 16 controls. The generated VCF file was filtered for structural variants on FCA B1, heterozygous in the standard Munchkin cats and homozygous wild type in the non-standard Munchkin cat and the 16 controls.

\section{Mutation analysis}

To test whether the $108 \mathrm{bp}$ insert is transcribed or not transcribed into mRNA, we isolated RNA from hair roots stabilized in RNAlater reagent (Qiagen, Hilden, Germany) of two standard Munchkin cats of the subtype Genetta and two domestic shorthair cats. All samples were transcribed into cDNA according to standard protocols (Maxima First Strand cDNA Synthesis Kit, Thermo Fisher). Primer pairs were designed using Primer3 tool (version 0.4.0, http://bioinfo.ut.ee/primer3-0.4.0/). One primer pair was designed to produce a PCR-product in mutant and wild type allele (PCR-type 1), one primer pair to produce a PCR-product only if the mutant allele is present and the insertion is transcribed (PCR-type 2), and one control primer pair to produce a PCR-product in the presence of wild type allele (PCR-type 3, Additional file 14). The first primer pair (PCR-type 1), located across the deletion from exon 10 (ENSFCAT00000009602.6) or exon 9 (ENSFCAT00000055794.2) to the 3'-UTR within the nondeleted region, yields an expected amplicon size of $510 \mathrm{bp}$ for the mutant allele if the insertion is transcribed and 402 bp for the mutant allele if the insertion is not transcribed, as well as $2514 \mathrm{bp}$ for the wild type allele (Additional file 7). The second primer pair (PCR-type 2) from the proximal region of exon 10 (ENSFCAT00000009602.6) or exon 9 (ENSFCAT00000055794.2), to a region within the $108 \mathrm{bp}$ insert produces an expected amplicon of $110 \mathrm{bp}$ only in the mutant allele when the insertion is transcribed. This PCRproduct is not present in the wild type or mutant allele when the insertion is not transcribed. The third primer pair (PCR-type 3) was designed to bridge exon 10 and 11 (ENSFCAT00000009602.6) or exon 9 and 10 (ENSFCAT00000055794.2) with an expected amplicon size of $185 \mathrm{bp}$ for the wild type allele in standard Munchkin cats and control cats with wild type alleles on both chromosomes. Furthermore, we designed five primer pairs (PCRtype 4-7) for cDNA sequencing the region spanning exon 10 (ENSFCAT00000009602.6) or exon 9 (ENSFCAT00000055794.2) and those parts of the 3'UTR present in the mutant and wild type allele (Additional file 15). PCR included $0.25 \mu \mathrm{M}$ of the respective primers, $20 \mathrm{ng}$ cDNA, $12.5 \mu \mathrm{l}$ UCP HiFidelity MasterMix (Qiagen, Hilden, Germany) and was prepared as recommended by Qiagen, Hilden, Germany. Sanger sequencing was done for the standard Munchkin dam from the Munchkin cat family of the subtype Genetta (Additional file 3) for PCR-type 1 and in one domestic shorthair cat for PCR-type 4-7.

In addition, open reading frames were predicted using ORF Finder (http://www.ncbi.nlm.nih.gov/projects/gorf/ ). Protein domains predicted by InterProScan (http:// www.ebi.ac.uk/interpro/search/sequence-search) for PANTHER [40] and PIRSF [41] were obtained by Ensembl database (protein summary) [42]. For comparative species alignments Clustal Omega (https://www.ebi. ac.uk/Tools/msa/clustalo/) was used [43]. Primer blast was done using NCBI nucleotide blast (https://blast.ncbi. nlm.nih.gov/Blast.cgi? $\quad$ PROGRAM = blastn\&PAGE TYPE $=$ BlastSearch\&LINK_LOC = blasthome). The sequence of the $108 \mathrm{bp}$ insertion was compared to the reference genomic sequences Felis catus 8.0 and Felis catus 9.0. The sequence similarity, highest alignment score and the expected value were calculated.

\section{Validation}

A structural variant composed of a $3303 \mathrm{bp}$ deletion (ss5015497294) and a 108 bp insertion (ss5015497295) within the critical region on FCA B1 was Sanger sequenced and visualized using a duplex PCR in individuals including standard Munchkin, non-standard Munchkin and control cats. Duplex PCR was performed in 260 individuals including 15 non-standard Munchkin, 42 standard Munchkin and 203 controls from breeds other than Munchkin cats. Sanger sequencing was done in the standard Munchkin sire, dam and the standard Munchkin kitten. A forward primer proximally of the detected structural variant and two different reverse primers located within the deleted region and distal of this deletion were designed using Primer3 tool (version 0.4.0, http:// bioinfo.ut.ee/primer3-0.4.0/) (Additional file 16 and 
Additional file 17). Duplex PCRs were performed in 22- $\mu \mathrm{l}$ reaction volumes containing $2 \mu \mathrm{l}$ DNA, $1.5 \mathrm{mM}$ deoxyribonucleoside triphosphates, 5 pmol of primers MK_del_R and MK_wt_R, 10 pmol of primer MK_wt_F, $1.5 \mathrm{U}$ of Taq polymerase in the reaction buffer supplied by the manufacturer (MP Biomedicals, Eschwege, Germany) and $4.2 \mu \mathrm{l}$ enhancer reagent (MP Biomedicals). After a $5 \mathrm{~min}$ initial denaturation at $95^{\circ} \mathrm{C}, 40$ cycles of $30 \mathrm{~s}$ at $94{ }^{\circ} \mathrm{C}, 30 \mathrm{~s}$ at $59^{\circ} \mathrm{C}$, and $45 \mathrm{~s}$ at $72{ }^{\circ} \mathrm{C}$ were run on a Thermocycler TProfessional 96 (Biometra, Göttingen, Germany). All samples were visually evaluated on a $1 \%$ agarose gel using Gel iX20 Imager (Intas Science Imaging Instruments, Göttingen, Germany).

Break point validation was performed in the three standard Munchkin cats of the subtype Genetta with WGS data using the primers MK_wt_F and MK_del_R accoding to the duplex PCR protocol. Evaluation of Sanger sequences was performed using Sequencher 4.8 software (GeneCodes, Ann Arbor, MI, USA).

\section{Supplementary information}

Supplementary information accompanies this paper at https://doi.org/10. 1186/s12863-020-00875-X.

Additional file 1. Survey on subtypes of Munchkin cats created by outcrossing with different cat breeds.

Additional file 2. Comparison of the length measurements for the long bones using CT images. A 4-year old male standard Munchkin cat is compared with an adult female domestic cat. Total (TL) and relative leg lengths, diaphyseal lengths (DL), and diaphyseal diameters (DD) in centimeters $(\mathrm{cm})$, and inches (in) of front and hind limbs are shown.

Additional file $\mathbf{3}$ Pedigree of Munchkin cat family of the subtype Genetta. Pedigree of the Munchkin cat family of the subtype Genetta segregating into long-legged and short-legged types. Furthermore, the pedigree shows that all standard Munchkin cats are heterozygous for the UGDH-indel (NC_018726.2:g.173294289_173297592delins108, Felis catus 8.0, equivalent to NC_018726.3:g.174882895_174886198delins108, Felis catus 9.0) and all typed non-standard Munchkin cats were homozygous wild type. For 32 cats, samples were not available and not typed.

Additional file 4 Case-control study for the standard Munchkin cat phenotype using 14 SNPs on feline chromosome B1. The SNPS B1_5 to B1_14 are located within the significantly genome-wide associated region at 168 to $184 \mathrm{Mb}$. SNP IDs with their nomenclature for Felis catus 8.0 and (Felis catus 9.0), odds ratios with their lower and upper confidence intervals $(C L), X^{2}$-square values for the genotypic and allelic distribution as well as the corresponding $-\log _{10} P$-values are shown. The most significant values are printed in bold.

Additional file 5 Haplotype analysis using SNPs B1_9 (g.173759872A > $\mathrm{G})$ and B1_10 (g.174407393 T > C) as well as the indel (NC_018726.2:g.173294289_173297592delins108) on feline chromosome B1 (Felis catus 8.0). The haplotypes with their frequencies, $X^{2}$-statistics and P-values are shown.

Additional file 6 Gene models of $U G D H$. UGDH has two transcripts, (A) $U G D H-201$ harboring 11 exons and (B) UGDH-202 harboring ten exons according to Ensembl for Felis catus 9.0. The detected UGDH-indel (NC_018726.2:g.173294289_173297592delins108, Felis catus 8.0, equivalent to NC_018726.3:g.174882895_174886198delins108, Felis catus 9.0) is causing a loss of $1191 \mathrm{bp}$ within the last intron, a complete loss of the last exon and a loss of 2001 bp of the 3'-UTR. Black boxes indicate exons. The chromosomal positions of the indel is given for Felis catus 8.0 reference genome (Felis catus 9.0 positions are in bold).
Additional file 7 Schematic representation of PCR-type 1-3 for CDNA in wild type (wt) and standard Munchkin cats (sMc) for (A) UGDH-201 and (B) UGDH-202 for Felis catus 9.0. The PCR-type 1 from exon 10 (ENSFCAT00000009602.6) or exon 9 (ENSFCAT00000055794.2) to the nondeleted 3'-UTR region should yield an expected amplicon size of $510 \mathrm{bp}$ for the mutant allele in case the insertion is transcribed and a 402 bp for the mutant allele when the insertion is not transcribed as well as $2514 \mathrm{bp}$ for the wild type allele. The PCR-type 2 from the proximal region of exon 10 (ENSFCAT00000009602.6) or exon 9 (ENSFCAT00000055794.2) to a region within the $108 \mathrm{bp}$ insert produces an expected amplicon size of $110 \mathrm{bp}$ for the mutant allele when the insertion is transcribed. PCR-type 3 from exon 10 to 11 (ENSFCAT00000009602.6) or exon 9 to 10 (ENSFCAT00000055794.2) produces an amplicon size of 185 bp in standard Munchkin cats and controls.

Additional file 8 DNA and CDNA sequences. The genomic DNA sequence of standard Munchkin cats (sMc) (A), the expected cDNA sequence based on this genomic DNA sequence (402 bp, B), as well as the validated Sanger sequence of CDNA with a confirmed amplicon size of $493 \mathrm{bp}$ (PCR-type 1, C) is shown. The $493 \mathrm{bp}$ amplicon comprises $73 \mathrm{bp}$ of the exon 10/9 (green), followed by a partially retained intron 10-11/910 of $309 \mathrm{bp}$ (blue) and $111 \mathrm{bp}$ of the $3^{\prime} \mathrm{UTR}$ (black). The $108 \mathrm{bp}$ insertion, which was not found in CDNA is given in pink. Start positions based on Felis catus 8.0 (Felis catus 9.0) and the newly predicted stop codon (underlined) are displayed.

Additional file $\mathbf{9}$ Variant effect on protein sequence. The amino acid (aa) sequence of the two transcripts (UGDH-201, ENSFCAT00000009602.6 and UGDH-202, ENSFCAT000000557945.1) with the newly predicted stop codon are shown. Open reading frame prediction gives a 12-aa truncated UGDH protein. Wild type cat (wt) protein sequences are compared to standard Munchkin cat (sMc) sequences.

Additional file 10 Domains predicted by InterProScan. (A) Predicted protein domains of transcript UGDH-201 (ENSFCAT00000009602.6) and (B) UGDH-202 (ENSFCAT000000557945.1) based on Felis catus 9.0 (Ensembl protein summary) are shown. Wild type cat (wt) protein length are compared to standard Munchkin cat (sMc) length.

Additional file 11. Comparative UGDH protein sequence alignment. Asterisks represent positions with a fully conserved residue, colons a conservation of strongly similar and periods weakly similar properties between different species. The indel is located within a highly conserved region of $U G D H$.

Additional file 12. Number of animals and breeds included in this study. Standard Munchkin cat subtypes, non-standard Munchkin cat subtypes and controls from various breeds are displayed.

Additional file $\mathbf{1 3}$ Primer pairs used for haplotype analysis. The single nucleotide variants located on chromosome B1 were genotyped using a Kompetitive Allele Specific PCR (KASP) assay. The SNP ID, nomenclature of Felis catus 8.0 and 9.0, accession numbers, sequences of forward and reverse primers, annealing temperature (AT), as well as the number of cycles are shown.

Additional file $\mathbf{1 4}$ Primer pairs used for complementary DNA amplification of UGDH. PCR was done to test if the $108 \mathrm{bp}$ insert is transcribed or not transcribed based on Felis catus 9.0. PCR-type 1 produces an expected amplicon of $510 \mathrm{bp}$ for the mutant allele if the insertion is transcribed, and a 402 bp for the mutant allele if the insertion is not transcribed, as well as 2514 bp for the wild type allele. PCR-type 2, spanning from exon 10 (UGDH-201 (ENSFCAT00000009602.6)) or exon 9 (UGDH-202 (ENSFCAT00000055794.2)) to the $108 \mathrm{bp}$ insert, should have an expected amplicon size of $110 \mathrm{bp}$ for the mutant allele if the insertion is transcribed. PCR-type 3 produces an amplicon with $185 \mathrm{bp}$ in standard Munchkin cats, and controls for the wild type allele.

Additional file 15. Primer pairs used for complementary DNA amplification spanning exon 10 (ENSFCAT00000009602.6) or exon 9 (ENSFCAT00000055794.2) to 3'UTR. All PCR-products were present in the wild type allele. PCR-type, primer pairs, sequences of forward and reverse primer, annealing temperatures (AT), and expected product sizes (bp) are shown. 
Additional file 16. Primer pairs used for validation and Sanger sequencing. Validation of the indel was done using duplex PCR (MK_wt_F, MK_wt_R and MK_del_R), amplicons for Sanger sequencing were generated with primer pairs MK_wt_F, and MK_del_R.

Additional file $\mathbf{1 7}$ Validation of deletion within $U G D H$ region (Felis catus 9.0). A forward primer (MK_wt_F) proximally of the detected structural variant and two different reverse primers located within the deleted region (MK_wt_R) and distal of this deletion (MK_del_R) are shown. An amplicon of primers MK_wt_F and MK_del_R could only be observed in mutant allele, whereas the PCR failed for the wild type allele. Primers MK_wt_F and MK_wt_R produced an amplicon only for the wild type allele.

\section{Abbreviations}

SNPs: Single nucleotide polymorphisms; FCA: Feline chromosome autosome; UGDH: UDP-Glucose 6-dehydrogenase; TICA: The International Cat Association; GWAS: Genome-wide association study; CT: Computed tomography; WGS: Whole genome sequencing; mm: Millimeter; In: Inch; Mb: Megabase; bp: Base pairs; UTR: Untranslated region; Indel: Insertiondeletion; aa: Amino acid; cDNA: Complementary DNA; ACAN: Aggrecan; HSPG: Heparan sulfate proteoglycan; UDP-GICA: UDP-glucuronic acid; Fgf: Fibroblast growth factor; Hh: Hedgehog; TGFß: Transforming growth factor- $\beta$; Wg,Wnt: Wingless; IACUC: The Institutional Animal Care and Use Committee; EDTA: Ethylenediaminetetraacetate; mAs: Milliampere seconds; kV: Kilovolt; DNA: Deoxyribonucleic acid; RNA: Ribonucleic acid; PCR: Polymerase chain reaction; mM: Millimolar; pmol: Picomol; KASP: Kompetitive Allele Specific PCR; ORF: Open reading frame finder; Min: Minute; TL: Total lengths; DL: Diaphyseal lengths; DD: Diaphyseal diameters; cm: Centimeters; wt: Wild type; sMc: Standard Munchkin cat

\section{Acknowledgements}

The authors are grateful to the owners of the cats donating samples and providing data for this research project. We thank J. Wrede for his help in bioinformatics and M. Drabert, H. Klippert-Hasberg, and N. Wagner for support in DNA preparation. We gratefully acknowledge The North-German Supercomputing Alliance (HLRN, Hannover) for providing high performance computing infrastructure and resources that contributed to the research results.

\section{Authors' contributions}

AS, JM and OD designed the study. AS and OD collected samples. AS, MB, KAD and JM performed sample preparation and carried out experiments. PD, $\mathrm{JN}$ and MF performed clinical examinations. AS and OD wrote the draft of the manuscript. OD performed bioinformatic analyses and completed the manuscript. All authors read and approved the final manuscript.

\section{Funding}

Not applicable.

\section{Availability of data and materials}

Variants were submitted to dbSNP database (http://www.ncbi.nlm.nih.gov/ SNP/) referred to as ss5015497294 and ss5015497295 (Felis catus 9.0; NC_018726.3:g. 174882895_174886198delins108). WGS data of the four Munchkin cats and eight controls were deposited in NCBI Sequence Read Archive (http://www.ncbi.nlm.nih.gov/sra) under the project number PRJNA505483 (SAMN10449164, SAMN10449165, SAMN10449169, SAMN10449170, SAMN14103114, SAMN14103116, SAMN14103119, SAMN14103120, SAMN14103121, SAMN14103123, SAMN14103124, SAMN14103127). Further WGS data for eight controls were retrieved from Sequence Read Archive (SRA, NCBI).

\section{Ethics approval and consent to participate}

All animal work was conducted according to the national and international guidelines for animal welfare. The present study had been specifically approved by the Institutional Animal Care and Use Committee (IACUC), the Lower Saxony state veterinary office at the Niedersächsisches Landesamt für Verbraucherschutz und Lebensmittelsicherheit, Oldenburg, Germany (registration number 33.9-42502-05-18A284).
Written informed approval was acquired from the cat owners and breeders to collect samples and measurements for current research, publication and further investigations.

\section{Consent for publication}

Not applicable.

\section{Competing interests}

The authors declare that they have no competing interests.

\section{Author details}

${ }^{1}$ Institute of Animal Breeding and Genetics, University of Veterinary Medicine Hannover (Foundation), 30559 Hannover, Germany. ${ }^{2}$ Clinic for Small Animals, University of Veterinary Medicine Hannover (Foundation), 30559 Hannover, Germany.

Received: 18 December 2019 Accepted: 24 June 2020

Published online: 30 June 2020

\section{References}

1. William-Jones $\mathrm{H}$. Arrested development of the long bones of the fore-limbs in a female cat. Vet Rec. 1944;56(449):131-2.

2. Vella CM, Shelton LM, McGonagle JJ, Stanglein TW. Robinson's genetics for cat breeders and veterinarians. 4th ed. Oxford: Butterworth-Heinemann Ltd; 1999.

3. Montavon PM, Voss K, Langley-Hobbs SJ. Feline orthopedic surgery and musculoskeletal disease. 1st ed. Edinburgh: Mosby; 2009.

4. Saben S. Munchkin cats and the munchkin cat. 1st ed. London, Great Britain: DYM Worldwide Publishers; 2016.

5. Bell J, Cavanagh K, Tilley L, Smith FW. Veterinary medical guide to dog and cat breeds. 1st ed. New York: CRC Press; 2012.

6. Registry RaEF. Jaguarette. 2019. https://rareandexoticfelinereg.homestead. com/breed_JGR.html. Accessed 13 Feb 2019.

7. Union IC. Cat breeds recognized in ICU. 2014. http://icun.ru/breed.html. Accessed 13 Feb 2018

8. Ann. The Munchkin Cat Scottish Fold Mix (The Scottish Kilt). 2019. https:// www.munchkincatguide.com/the-munchkin-cat-scottish-fold-mix-thescottish-kilt/. Accessed 16 Aug 2019.

9. Pet P. Gaelic folds are the best of both breeds. 2023. https://kdunnetsmith wixsite.com/nowstarring. Accessed 13 Feb 2019.

10. C D. 8 Insanely Adorable Munchkin Cat Breeds That Will Melt Your Heart. 2017. https://catvills.com/8-munchkin-cat-breeds/. Accessed 13 Feb 2019.

11. Andersen L-A. Ultimate Cat: A Complete Guide to Breeds and Hybrids. 1st ed. Morrisville: Tear Drop Books; 2016.

12. TICA. Munchkin Breed 2018 https://www.tica.org/breeds/browse-allbreeds?view=article\&id=857: munchkin-breed\&catid=79. Accessed 13 Feb 2018.

13. Sellers S, Gandolfi B, Gustafson N, Coates J, Fox D, Britt L, et al. The Munchkin cat: Phenotypic and Genotypic Characterization. 2014. Accessed 13 Feb 2018. http://cvm.missouri.edu/vrs/2014VRSPposters/pdfs/Sellers.pdf.

14. Lander AD, Selleck SB. The elusive functions of proteoglycans: in vivo veritas. J Cell Biol. 2000;148(2):227-32.

15. The I, Bellaiche $Y$, Perrimon N. Hedgehog movement is regulated through tout velu-dependent synthesis of a heparan sulfate proteoglycan. Mol Cell. 1999;4(4):633-9.

16. Bernfield M, Götte M, Park PW, Reizes O, Fitzgerald ML, Lincecum J, et al. Functions of cell surface heparan sulfate proteoglycans. Annu Rev Biochem. 1999:68(1):729-77.

17. Weaver CM, Fuchs RK. Skeletal growth and development. Basic and Applied Bone Biology. Cambridge: Elsevier; 2014. p. 245-60.

18. Hartmann C, Tabin CJ. Dual roles of Wnt signaling during chondrogenesis in the chicken limb. Development. 2000;127(14):3141-59.

19. Norton WH, Ledin J, Grandel H, Neumann CJ. HSPG synthesis by zebrafish Ext2 and Ext13 is required for Fgf10 signalling during limb development. Development. 2005;132(22):4963-73.

20. Rudnicki JA, Brown AM. Inhibition of Chondrogenesis by Wnt gene expression in vivo and in vitro. Dev Biol. 1997;185(1):104-18.

21. Metzger J, Wöhlke A, Mischke R, Hoffmann A, Hewicker-Trautwein M, Küch E-M, et al. A novel SLC27A4 splice acceptor site mutation in great Danes with ichthyosis. PLoS One. 2015;10(10):e0141514. 
22. Singh RK, Cooper TA. Pre-mRNA splicing in disease and therapeutics. Trends Mol Med. 2012;18(8):472-82.

23. Gontijo AM, Miguela V, Whiting MF, Woodruff R, Dominguez M. Intron retention in the Drosophila melanogaster Rieske Iron Sulphur protein gene generated a new protein. Nat Commun. 2011;2:323.

24. Struck A-K, Dierks C, Braun M, Hellige M, Wagner A, Oelmaier B, et al. A recessive lethal chondrodysplasia in a miniature zebu family results from an insertion affecting the chondroitin sulfat domain of aggrecan. BMC Genet. 2018;19(1):91

25. Cavanagh JA, Tammen I, Windsor PA, Bateman JF, Savarirayan R, Nicholas FW, et al. Bulldog dwarfism in Dexter cattle is caused by mutations in ACAN. Mamm Genome. 2007;18(11):808-14.

26. Lin X, Wei G, Shi Z, Dryer L, Esko JD, Wells DE, et al. Disruption of gastrulation and heparan sulfate biosynthesis in EXT1-deficient mice. Dev Biol. 2000:224(2):299-311.

27. Topczewski J, Sepich DS, Myers DC, Walker C, Amores A, Lele Z, et al. The zebrafish glypican knypek controls cell polarity during gastrulation movements of convergent extension. Dev Cell. 2001;1 (2):251-64.

28. Walsh EC, Stainier DY. UDP-glucose dehydrogenase required for cardiac valve formation in zebrafish. Science. 2001;293(5535):1670-3.

29. García-García MJ, Anderson KV. Essential role of glycosaminoglycans in Fgf signaling during mouse gastrulation. Cell. 2003;114(6):727-37.

30. Hacker U, Lin X, Perrimon N. The Drosophila sugarless gene modulates wingless signaling and encodes an enzyme involved in polysaccharide biosynthesis. Development. 1997;124(18):3565-73.

31. Jones JM, Jolly R. Dwarfism in Hereford cattle: a genetic morphological and biochemical study. N Z Vet J. 1982;30(12):185-9.

32. Andrews S. FastQC: a quality control tool for high throughput sequence data; 2010.

33. Schmieder R, Edwards R. Quality control and preprocessing of metagenomic datasets. Bioinformatics. 2011;27(6):863-4.

34. Li H, Durbin R. Fast and accurate short read alignment with burrowswheeler transform. Bioinformatics. 2009:25(14):1754-60

35. Li H, Handsaker B, Wysoker A, Fennell T, Ruan J, Homer N, et al. The sequence alignment/map format and SAMtools. Bioinformatics. 2009;25(16): 2078-9.

36. McKenna A, Hanna M, Banks E, Sivachenko A, Cibulskis K, Kernytsky A, et al. The genome analysis toolkit: a MapReduce framework for analyzing nextgeneration DNA sequencing data. Genome Res. 2010;20(9):1297-303.

37. DePristo MA, Banks E, Poplin R, Garimella KV, Maguire JR, Hartl C, et al. A framework for variation discovery and genotyping using next-generation DNA sequencing data. Nat Genet. 2011;43(5):491

38. Cingolani P, Platts A, Wang LL, Coon M, Nguyen T, Wang L, et al. A program for annotating and predicting the effects of single nucleotide polymorphisms, SnpEff: SNPs in the genome of Drosophila melanogaster strain w1118; iso-2; iso-3. Fly. 2012;6(2):80-92.

39. Layer RM, Chiang C, Quinlan AR, Hall IM. LUMPY: a probabilistic framework for structural variant discovery. Genome Biol. 2014;15(6):R84.

40. Mi H, Lazareva-Ulitsky B, Loo R, Kejariwal A, Vandergriff J, Rabkin S, et al. The PANTHER database of protein families, subfamilies, functions and pathways. Nucleic Acids Res. 2005;33(suppl_1):D284-D8.

41. Wu CH, Nikolskaya A, Huang H, Yeh LSL, Natale DA, Vinayaka CR, et al. PIRSF: family classification system at the Protein Information Resource. Nucleic Acids Res. 2004;32(suppl_1):D112-D4.

42. Quevillon E, Silventoinen V, Pillai S, Harte N, Mulder N, Apweiler R, et al. InterProScan: protein domains identifier. Nucleic Acids Res. 2005;33(suppl_ 2):W116-W20

43. Sievers F, Wilm A, Dineen D, Gibson TJ, Karplus K, Li W, et al. Fast, scalable generation of high-quality protein multiple sequence alignments using Clustal omega. Mol Syst Biol. 2011;7(1):539.

\section{Publisher's Note}

Springer Nature remains neutral with regard to jurisdictional claims in published maps and institutional affiliations.

Ready to submit your research? Choose BMC and benefit from:

- fast, convenient online submission

- thorough peer review by experienced researchers in your field

- rapid publication on acceptance

- support for research data, including large and complex data types

- gold Open Access which fosters wider collaboration and increased citations

- maximum visibility for your research: over $100 \mathrm{M}$ website views per year

At BMC, research is always in progress.

Learn more biomedcentral.com/submissions 